\title{
Evaluation of Different Methods for Voltage Sag Source Detection Based on Positive-Sequence Components
}

\author{
Boštjan Polajžer, Gorazd Štumberger, Drago Dolinar \\ University of Maribor \\ Faculty of Electrical Engineering and Computer Science \\ Smetanova 17, 2000 Maribor, Slovenia \\ phone:+386 2220 7076, fax:+386 2220 7272, e-mail: bostjan.polajzer@uni-mb.si
}

\begin{abstract}
This paper evaluates methods for voltage sag source detection, which are based either on energy, current or impedance criteria. It is shown that some methods known from the literature do not work well, particularly in cases of asymmetrical voltage sags. Furthermore, some methods require measurements of instantaneous values, which is not always feasible. Therefore, symmetrical component transformation is applied, whereas only positive-sequence components are used. All the discussed methods were tested by applying extensive simulations and field tests. The obtained results show that all methods which are based on positive-sequence components are highly effective also in cases of asymmetrical voltage sags.
\end{abstract}

Key words power systems, power quality, voltage sag, source detection.

\section{Introduction}

Voltage sags are common power-quality disturbances which may, despite their relatively short duration, be detrimental to several industrial loads. Furthermore, voltage sags are rather frequent, since they can be provoked by different events throughout the network, such as faults, motor starting, transformer energizing and heavy load switching [1]. Disturbances caused by voltage sags influence the production losses of customers and the utility [2]. In such cases, any disputes can only be resolved fairly if the voltage sag source is reliably detected. Even though a methodology for pinpointing the exact locations of voltage sags does not yet exist, several methods for voltage sag source detection have already been reported [3]-[7].

This paper discusses four different methods for voltage sag source detection. The method proposed in [3] is based on the assumption that energy flow at the monitoring point changes during voltage sags. Thus, disturbance energy is used as the criterion. The methods proposed in [4],[5] are both based on the assumption that currents measured at the monitoring point change during the voltage sag. The slope of a voltage-current characteristic is investigated in [4], whereas the time behavior of an active current component is observed in [5]. Criteria within these two methods are both checked for each phase individually. In the cases of asymmetrical voltage sags, individual phases show different behavior. In such cases the usage of positivesequence components may be helpful, yet in [4],[5] this is not suggested. In [6] an impedance-based method is proposed, where the real part of the estimated positivesequence impedance is used to determine the direction of the voltage sag source.

Methods proposed in [4]-[6] all require calculation of phasors for fundamental-frequency component. Due to the averaging in the harmonic analysis of the input signals these methods might produce questionable results. Methods that are based on instantaneous values [3],[7] are expected to give more reliable results. However, measurement of instantaneous values is not always feasible. Most power-quality monitors, namely, provide us with information on rms values, power factor, harmonics, symmetrical components, etc. The discussed methods are, therefore, adopted to work with the positive-sequence components. All the discussed methods for voltage sag source detection were tested by applying extensive simulations and field tests.

\section{Methods for voltage sag source detection based on positive-sequence components}

Let us consider the system shown in Fig. 1. A recording device is placed at the monitoring point (MP). Line voltages and currents have to be recorded in order to determine on which side of the recording device the voltage sag originated. In regard to the pre-sag energy flow direction, upstream and downstream events are defined in points A and B, respectively.

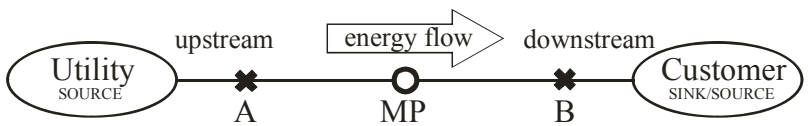

Fig. 1. Upstream event at the utility side (A) and downstream event at the customer side (B) 


\section{A. Symmetrical Component Transformation}

Fundamental-frequency voltage and current phasors $\underline{U}_{k}$ and $\underline{I}_{k}$, are transformed by (1), where $k \in\{a, b, c\} \quad(a, b, c$ denote individual phases). $\mathbf{F}$ is the unitary symmetrical component (Fortescue's) transformation matrix (2), where * denotes the complex conjugate. In this way voltage and current phasors $\underline{U}^{+}, \underline{I}^{+}, \underline{U}^{-}, \underline{I}^{-}$and $\underline{U}^{0}, \underline{I}^{0}$ are obtained for positive, negative and zero-sequencecomponents, respectively.

$$
\begin{gathered}
{\left[\begin{array}{l}
\underline{U}^{+} \\
\underline{U}^{0}
\end{array}\right]=\mathbf{F}\left[\begin{array}{l}
\underline{U}^{a} \\
\underline{U}_{b}^{b} \\
\underline{U}_{c}
\end{array}\right],\left[\begin{array}{c}
\underline{I}^{+} \\
\underline{I}^{0} \\
\underline{I}^{0}
\end{array}\right]=\mathbf{F}\left[\begin{array}{c}
\underline{I}_{I} a \\
\underline{I}_{c}
\end{array}\right]} \\
\mathbf{F}=\frac{1}{\sqrt{3}}\left[\begin{array}{ccc}
1 & e^{j 2 \pi / 3} & e^{j 4 \pi / 3} \\
1 & e^{j 4 \pi / 3} & e^{j 2 \pi / 3} \\
1 & 1 & 1
\end{array}\right], \quad \mathbf{F}^{-1}=\left(\mathbf{F}^{*}\right)^{T}
\end{gathered}
$$

Active power is expressed by (3), where $P^{+}, P^{-}$and $P^{0}$ are the positive, negative and zero-sequence active power, respectively.

$$
P=\operatorname{Re}\left(\underline{U}^{+} \underline{I}^{+^{*}}+\underline{U}^{-} \underline{I}^{-^{*}}+\underline{U}^{0} \underline{I}^{0^{*}}\right)=P^{+}+P^{-}+P^{0}
$$

\section{B. Energy-Based Method}

Voltage sags are provoked by different events which can all be treated as energy sinks. Thus, it can be assumed that the energy flow at the monitoring point increases during downstream events and decreases during upstream events. In order to detect the voltage sag source, the disturbance energy $\Delta w(t)$ is used as the criterion (4)-[3].

$$
\Delta w(t)=\int_{0}^{t}\left(p_{\text {sag }}(\tau)-p_{\text {presag }}(\tau)\right) d \tau\left\{\begin{array}{l}
<0 \Rightarrow \text { upstream } \\
>0 \Rightarrow \text { downstream }
\end{array}\right.
$$

This method requires measurement of instantaneous values, which is not always feasible. Therefore, the positive-sequence active power $P^{+}$is used in (4). In this way new energy-based method is introduced.

\section{Voltage-Current Method}

Voltage sags are due to short-duration increases in currents elsewhere in the network. Thus, currents measured at the monitoring point increase during downstream events and decrease during upstream events, as shown in Fig. 2.

a)

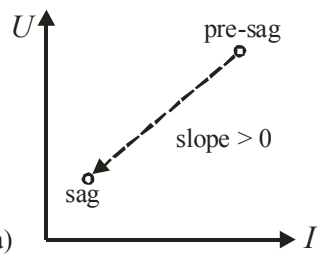

Fig. 2. $U-I$ characteristics in case of upstream event (a) and downstream event (b)
Based on this assumption the phasor-based voltagecurrent method is proposed in [4]. Once the voltage sag is captured points of the characteristic $\left(\left|\underline{I}_{k}\right|,\left|\underline{U}_{k} \cos \phi_{k}\right|\right)$ are approximated using the linear function in order to investigate the slope (5). $\left|\underline{U}_{k}\right|$ and $\left|\underline{I}_{k}\right|$ are lengths of the voltage and current phasor, $\phi_{k}$ is a phase angle.

$$
\text { slope }\left(\left|\underline{I}_{k}\right|,\left|\underline{U}_{k} \cos \phi_{k}\right|\right)\left\{\begin{array}{l}
>0 \Rightarrow \text { upstream } \\
<0 \Rightarrow \text { downstream }
\end{array}\right.
$$

The criterion (5) is checked for each phase individually. Consequently, the obtained results may not be interpreted in a unique way, especially in the cases of asymmetrical voltage sags. Symmetrical component transformation is, therefore, applied. New voltage-current method is introduced in such a way that positive-sequence voltage and current phasors $\underline{U}^{+}$and $\underline{I}^{+}$are used in (5).

\section{Active Current-Based Method}

On the same assumptions as the voltage-current method [4] another method is proposed in [5]. Within this method the time response of an active current component $\left(\left|I_{k}\right| \cos \phi_{k}\right)$ is calculated for a few cycles prior and during the voltage sag. The sign of its first peak at the beginning of the voltage sag is used as the criterion (6). $\left|\underline{I}_{k}\right|$ is a current phasor length, whereas $\phi_{k}$ is a phase angle.

$$
\text { first peak }\left(\left|\underline{I}_{k}\right| \cos \phi_{k}\right)\left\{\begin{array}{l}
<0 \Rightarrow \text { upstream } \\
>0 \Rightarrow \text { downstream }
\end{array}\right.
$$

This criterion is also checked for each phase individually. In order to obtain conclusive results in the cases of asymmetrical voltage sags, the positive-sequence voltage and current phasors $\underline{U}^{+}$and $\underline{I}^{+}$are used in (6). In this way new active current-based method is introduced.

\section{E. Impedance-Based Method}

The concept of incremental impedance is proposed in [6], which is negative for downstream events and positive for upstream events. However, only the real part of the estimated positive-sequence impedance is used as the criterion (7). Incremental impedance is obtained from the ratio $\Delta \underline{Z}^{+}=\left(\Delta U^{+} / \Delta I^{+}\right)$, where the incremental voltage is defined by $\Delta \underline{U}^{+}:=\left(\underline{U}^{+}\right.$sag $-\underline{U}^{+}$presag $)$, whereas the incremental current by $\Delta \underline{I}^{+}:=\left(\underline{I}^{+}\right.$sag $-\underline{I}^{+}$presag $)$.

$$
\operatorname{Re}\left(\Delta \underline{Z}^{+}\right)=\Delta R^{+} \begin{cases}<0 & \Rightarrow \text { upstream } \\ >0 & \Rightarrow \text { downstream }\end{cases}
$$

In order to improve the impedance estimation, adaptive multiple-cycles of data are used by applying the least squares method. However, this method can produce unreliable results in cases of voltage sags provoked by upstream faults in a radial network. In such cases, namely, there might be no change in seen impedance, neither in magnitude nor in angle. 


\section{Results}

\section{A. Numerical simulations}

A radial and non-radial testing network (Fig. 3) was used for numerical simulations of voltage sags. An extensive number of tests were performed using MATLAB/Simulink/SimPowerSystems. Different types of loads, such as RL-load, induction motor, synchronous motor, as well as constant power loads were used in different combinations. In order to simulate the distributed generation small induction and synchronous generators were also used. Four types of faults were applied in four different locations (FL1-FL4): phase-toground (P-G), phase-to-phase-to-ground (P-P-G), phaseto-phase (P-P) and three-phase (3-P). In this way voltage sags were generated with a sufficient magnitude $(>15 \%)$ and duration of $100 \mathrm{~ms}$. Furthermore, voltage sags due to heavy motor starting and loading were also simulated. Voltages and currents were captured at four monitoring points (MP1-MP4). Harmonics and measurement noise were not taken into account.

Examples of the simulation results are shown in Figs. 4 and 5. In Fig. 4 results are shown for the upstream P-G fault in a radial network at location FL2. Voltages and currents were captured at terminals of a RL-load, that is, at MP4 (Fig. 3). Considerable asymmetry in rms voltages typical for P-G faults is shown in Fig. 4a. The original energy-based method incorrectly indicated downstream event $(\Delta w>0$ - Fig. $4 \mathrm{~b})$. When the positive-sequence power was used the result was correct $(\Delta w<0)$, that is upstream. Results obtained by the voltage-current method (Fig. 4c) are correct regardless the quantities being used (phase or positive-sequence). Positive slopes namely indicate an upstream event. From results obtained by the active-current based method (Fig. 4d) it is obvious that individual phases show different behavior. When positive-sequence components were used the obtained result was correct (negative first peak), however a quite small change was noticed in the observed time response. Result obtained with the impedance-based method was correct $(+1.6 \Omega)$, yet inconclusive, since the resultant resistance did not change during the sag.

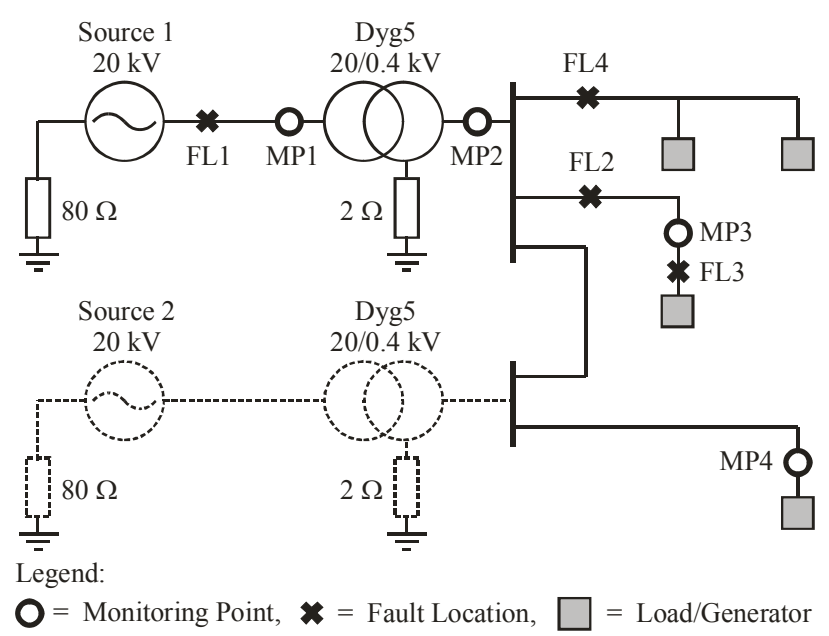

Fig. 3. Testing-network for simulations of voltage sags: radial network (solid line) and non-radial network (solid and dashed line)
In Fig. 5 results are shown for an example of a P-P fault at location FL3. Voltages and currents were captured at MP4, that is on terminals of a small induction generator which was placed in a radial network to simulate distributed generation. Thus, the voltage sag originated on the downstream side of MP4 (Fig. 3). Even though captured at terminals of an induction generator, considerable voltage sag with the magnitude of $25 \%$ can be noticed in phase $b$ (Fig. 5a). Results obtained by the energy-based method (Fig. 5b) were correct regardless the quantities being used (instantaneous or positivesequence) since in both cases a downstream event was indicated $(\Delta w>0)$. On the contrary, results obtained by the voltage-current method and active current method (Figs. 5c and 5d) were inconclusive, since they show different behavior for different phases. However, when the positive-sequence components were used then the obtained results correctly indicated a downstream event, that is negative slope within the voltage-current method (Fig. 5c) and positive first peak within the active currentbased method (Fig. 5d). The impedance-based method correctly indicated a downstream event since the obtained incremental resistance was negative $(-0.1 \Omega)$.
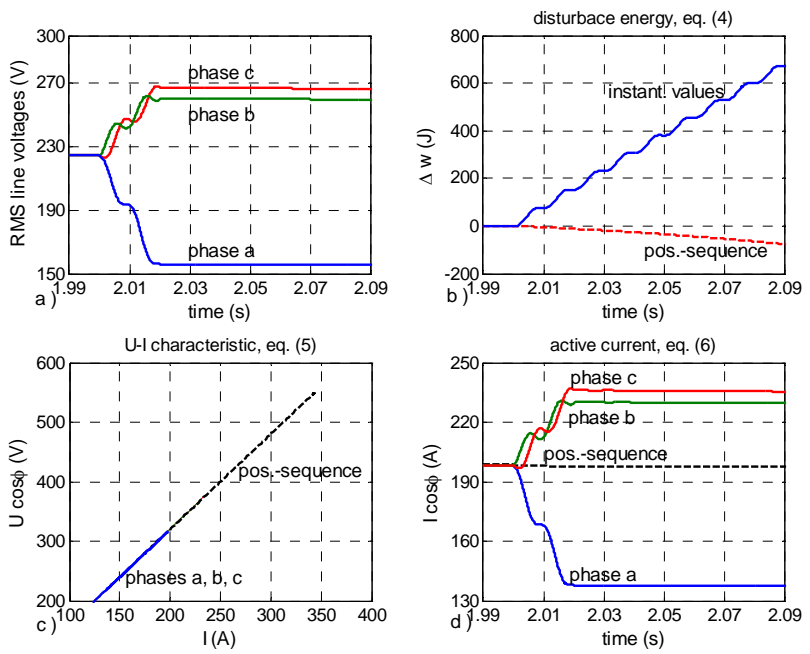

Fig. 4. Simulation results for the upstream phase-to -ground fault, $\Delta R^{+}=+1.6 \Omega$ yet not changed
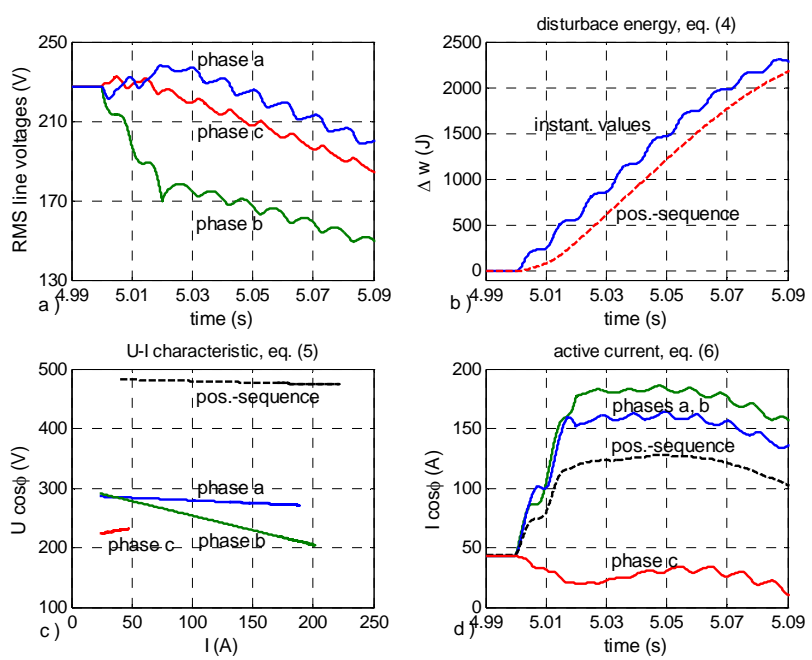

Fig. 5. Simulation results for the downstream phase-to-phase fault, $\Delta R^{+}=-0.1 \Omega$ 


\section{B. Field testing}

An extensive number of field tests were also applied to all the discussed methods. Voltages and currents were captured in Slovenian power system at different voltage levels $(400 \mathrm{kV}, 220 \mathrm{kV}, 110 \mathrm{kV}, 20 \mathrm{kV}$ and $0.4 \mathrm{kV})$, whereas voltage sags were provoked by different events, such as 3-P faults, P-P faults, P-G faults and transformer energizing.

An example of field testing results is shown in Fig 6. A downstream voltage sag was provoked by a P-P fault on the primary side terminals of a distribution transformer $20 / 0.4 \mathrm{kV}$. Voltage sag with the magnitude of $10 \%$ (Fig. 6a) was captured at the nearest $20 \mathrm{kV}$ bus. All the discussed methods, except the voltage-current method, correctly indicated a downstream event $(\Delta w>0$, current peak $>0, \Delta R^{+}<0$ ), as shown in Figs. $6 \mathrm{~b}$ and $6 \mathrm{~d}$. Result obtained using the current-based method was inconclusive since individual phases show different slopes (Fig. 6c). However, when the positive-sequence components were applied, the result obtained by the voltage-current method was also correct (slope $<0$ ).
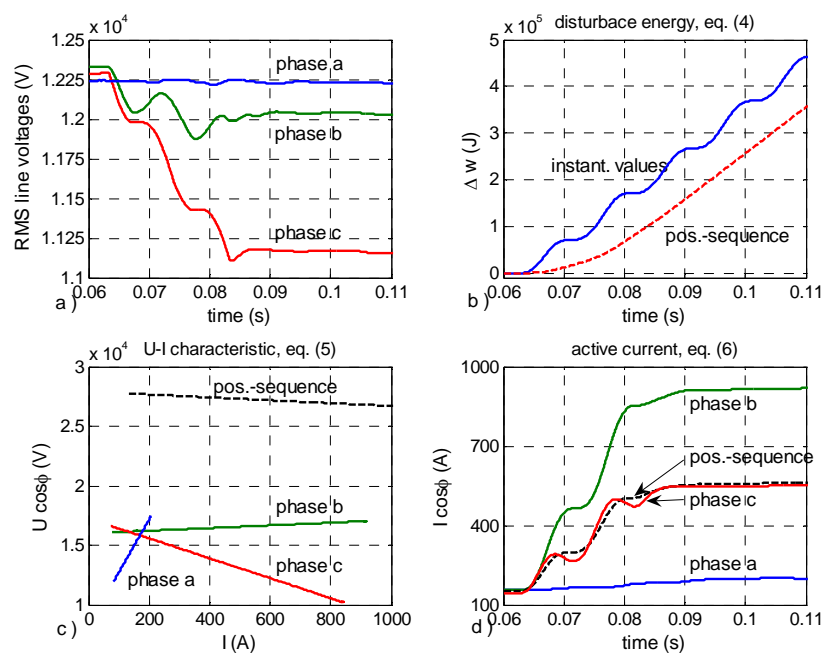

Fig. 6. Filed testing results for the downstream phase-to-phase fault, $\Delta R^{+}=-3.6 \Omega$

\section{Evaluation of discussed methods for voltage sag source detection}

All the discussed methods for voltage sag source detection were tested for altogether 194 various examples of voltage sags (174 simulations and 20 field tests). Symmetrical voltage sags due to 3-P faults, motor loading and motor starting were separately examined (50 examples), as well as asymmetrical voltage sags due to P-G faults, P-P-G faults, P-P faults, and transformer energizing (144 examples). Special attention was paid also to voltage sags due to P-G faults (97 examples), since they are the most frequent ones. Effectiveness was calculated for all the discussed methods. The obtained results are shown in Fig. 7.

The energy-based method shows extremely high effectiveness in the cases of symmetrical and asymmetrical voltage sags (Fig. 7a). Moreover, when the positive-sequence active power was applied the results were correct in all 194 examples. The original method gave us incorrect results only in some cases of P-G faults, where source impedance was relatively small. A disturbing effect of the zero-sequence power is thus more than evident, which is discussed also in [7].

The original voltage-current and active current-based methods, on the contrary, show very low effectiveness (Figs. $7 \mathrm{~b}$ and 7c) - in cases of asymmetrical voltage sags even less than $50 \%$. Thus, these two methods can not be used in cases of asymmetrical voltage sags. When the positive-sequence quantities were applied to the voltagecurrent method, then this method was indeed more effective (approx. 70\% - Fig. 7b), yet not enough to obtain reliable results. However, when the positivesequence quantities were used within the active-current based method, then the effectiveness of this method was increased up to $94 \%$ (Fig. 7c).

The impedance-based method shows quite low effectiveness (approx. 70\% - Fig. 7d). This is mostly due to the inconclusive results in cases of voltage sags provoked by upstream faults where the seen impedance was not changed.

Note, that all the discussed methods show almost the same effectiveness for voltage sags captured in the radial network and those captured in the non-radial network.
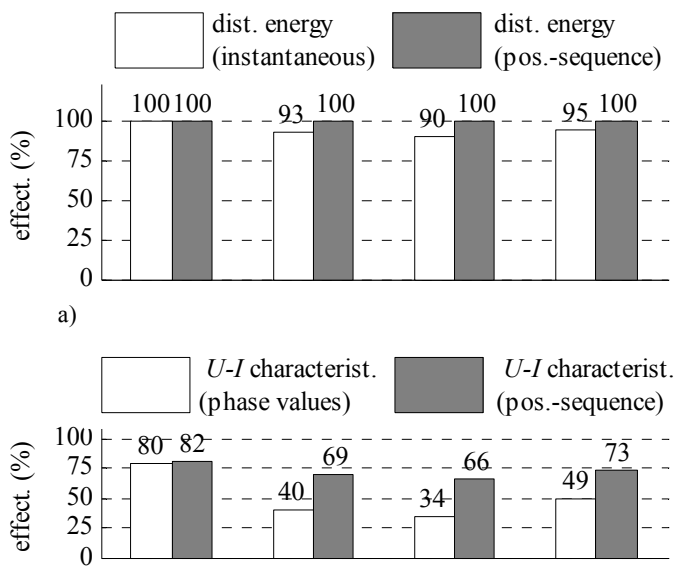

b)

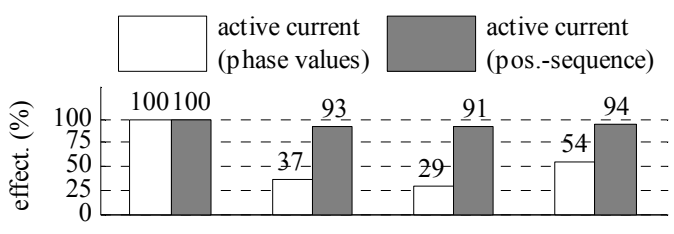

c)

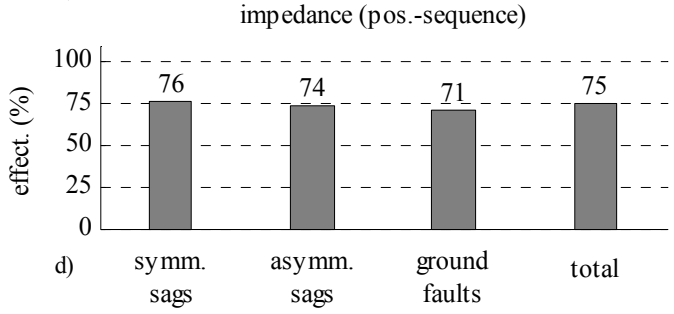

Fig. 7. Effectiveness of the discussed methods: energy-based (a), voltage-current (b), active current-based (c), and impedance based (d) 


\section{Conclusion}

This work discusses different methods for voltage sag source detection. Symmetrical component transformation is applied to the already known methods, thus three new methods are introduced by considering only positivesequence components. Since all the proposed methods require calculations of phasors for the fundamentalfrequency, the obtained results might be affected by harmonics, transients and measurement noise in the input signals. However, in the cases where measurement of instantaneous values is not feasible, applying the positive-sequence components might be the only reasonable solution. All the discussed methods for voltage sag source detection were tested using extensive simulations and field testing. Based on the performed evaluation results it can be concluded that in the cases of asymmetrical voltage sags the proposed methods are much more effective than the original methods, which was completely confirmed by field testing results.

\section{Acknowledgement}

This work was supported by ARRS, Projects no. P2-0115 and L2-7560-1792.

\section{References}

[1] M. J. H. Bollen, "Voltage sags in three-phase systems," IEEE Power Engineering Review, vol. 21, no. 9, pp.8-11,15, September 2001.

[2] C. J. Melhorn, T. D. Davis and G. E. Beam, "Voltage sags: their impact on the utility and industrial customers," IEEE Transactions on Industry Applications, vol. 34, no. 3, pp. 549558, May-June 1998.

[3] A. C. Parsons, W. M. Grady, E. J. Powers and J. C. Soward, "A direction finder for power quality disturbances based upon disturbance power and energy," IEEE Transactions on Power Delivery, vol. 15, no. 3, pp. 1081-1086, July 2000.

[4] C. Li, T. Tayjasanant, W. Xu and X. Liu, "Method for voltagesag-source detection by investigating slope of the system trajectory," IEE Proceedings: Generation, Transmission and Distribution, vol. 150, no. 3, pp. 367-372, May 2003.

[5] N. Hamzah, A. Mohamed and A. Hussain, "A new approach to locate the voltage sag source using real current component," Electric Power Systems Research, vol. 72, no. 2, pp. 113-123, December 2004.

[6] T. Tayjasanant,V. Li, and W. Xu, "A resistance sign-based method for voltage sag source detection," IEEE Transactions on Power Delivery, vol. 20, no. 4, pp. 2544-2551, October 2005.

[7] B. Polajžer, G. Štumberger, S. Seme, and D. Dolinar, "Detection of voltage sag sources based on instantaneous voltage and current vectors and orthogonal Clarke's transformation," IET Proceedings: Generation, Transmission and Distribution, vol. 2, no. 2, pp. 219-226, March 2008. 